\title{
Posterior compartment syndrome following ruptured Baker's cyst*
}

\author{
David P Petros, James F Hanley, Patricia Gilbreath, Robert D Toon
}

\begin{abstract}
A 35 year old man presented with the sudden onset of left leg pain and swelling. Empirical anticoagulation for presumed deep vein thrombophlebitis was followed by acute worsening of symptoms. On further evaluation the patient was found to have a ruptured Baker's cyst and an acute posterior compartment syndrome.
\end{abstract}

Popliteal or Baker's cyst have been well described in medical reports. ${ }^{1-4}$ Acute rupture has received special consideration owing to the dramatic presentation and the confusion it may create with deep vein thrombophlebitis. We present a case of a ruptured Baker's cyst complicated by an acute posterior compartment syndrome.

\section{Case report}

A 35 year old, previously healthy man presented to an outlying hospital two weeks before admission with pain and swelling in his left leg. No specific cause was found, and the patient was treated with a compressive dressing and advised to limit his activities. Despite initial mild improvement he presented to Landstuhl Army Regional Medical Center-with acute worsening of his pain and swelling after feeling a 'pop' in the proximal left calf. There was no past history of joint disease, no trauma to the affected leg, or excessive physical activity preceding the onset of symptoms.

On physical examination the left calf was swollen, tender, and warm. The circumference of the left calf was $44 \mathrm{~cm}$ compared with $42 \mathrm{~cm}$ on the right. The range of motion in the left knee was mildly decreased secondary to a small effusion.

The patient was admitted late in the evening with a presumptive diagnosis of left calf deep vein thrombophlebitis, and treatment with intravenous heparin was begun. By the next morning the patient had increasing pain. The circumference of the left calf now measured 47 cm. A venogram showed a complete cut off at the popliteal space (fig 1), most consistent with external compression. Heparin was discontinued. A computed tomographic scan was unremarkable except for swelling of the muscle bundles and soft tissue. Arthrocentesis on the left knee removed $20 \mathrm{ml}$ of bloody fluid. Microscopic examination showed numerous erythrocytes, few neutrophils, and no crystals. An arthrogram showed extravasation of dye posteriorly into the calf (fig 2).

After the arthrogram the patient continued to

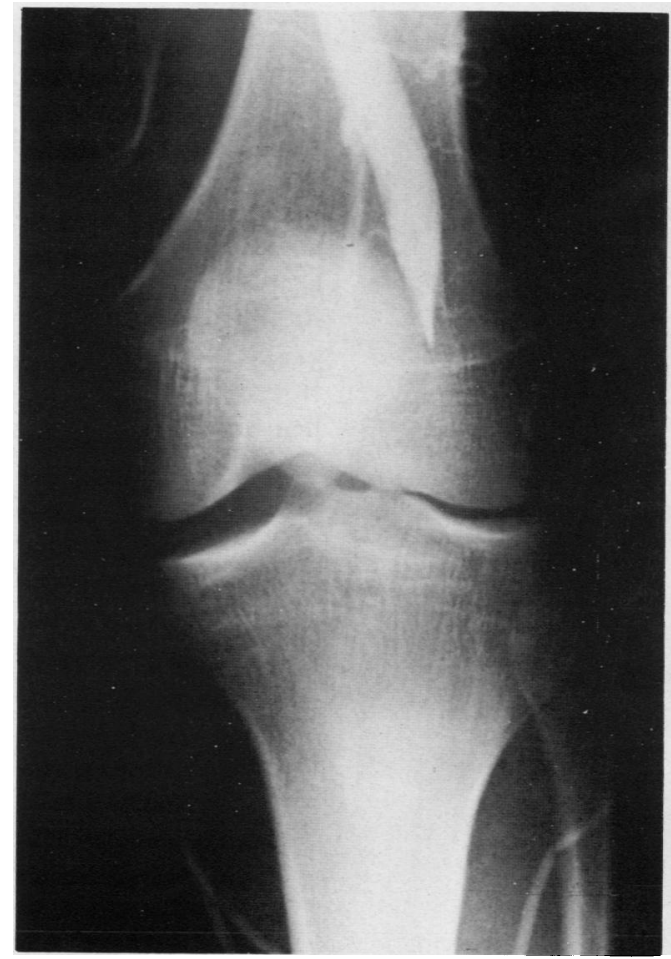

Figure 1 Venogram showing complete cut off of the popliteal vein at the popliteal space.

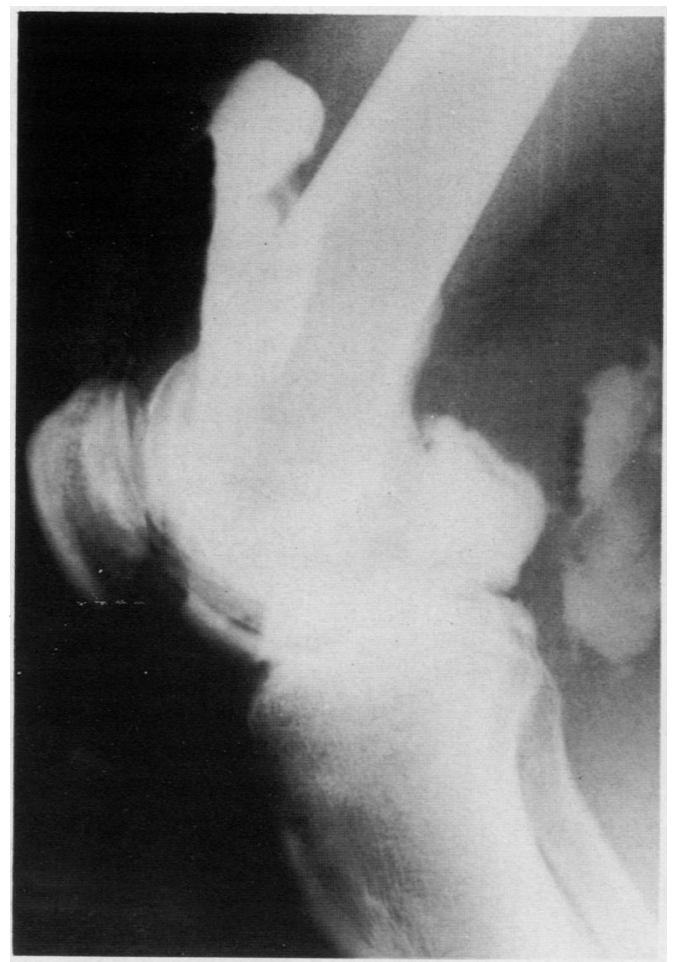

Figure 2 Arthrogram showing posterior extravasation of contrast with extension into the calf. 
have severe pain in the calf. Examination showed decreased sensation in the left foot with intact motor function. Anterior, lateral, and superficial posterior compartment pressures by the infusion technique were 58,59 , and 81 $\mathrm{mmHg}$ respectively, on the left compared with $27 \mathrm{mmHg}$ in the right superficial posterior compartment. A four compartment fasciotomy was performed disclosing haemorrhage in the posteromedial gastrocnemius muscle and diffuse swelling. After surgery a repeat venogram was normal. The patient's subsequent recovery was prolonged, requiring delayed primary closures over the following month. Four months later the patient has slight numbness in the distribution of the left saphenous nerve and wears a support stocking for persistent dependent oedema. The left knee is normal.

\section{Discussion}

Popliteal or Baker's cysts were initially described by Adams in $1840^{1}$ and later in more detail by Baker in $1877 .^{2}$ Most authors agree they represent distended gastrocnemio-semimembranosus bursae. ${ }^{34}$ Any intra-articular process associated with effusions may lead to distension of this bursa through a one-way valve mechanism. Intra-articular pressures in patients with knee effusions as high as $1000 \mathrm{mmHg}$ with the knee completely bent have been reported. Although most Baker's cysts remain asymptomatic or are associated with only mild localised symptoms, a variety of complications have been reported. The most dramatic is acute rupture with the clinical presentation often called the pseudothrombophlebitis syndrome. ${ }^{5}$

Our patient presented with an acutely ruptured Baker's cyst. The minimal symptomatology and physical findings of the knee on admission clouded the correct diagnosis. Empirical treatment with intravenous heparin for presumed deep vein thrombophlebitis might have precipitated the compartment syndrome. The acute worsening of symptoms after treatment with heparin, the bloody arthrocentesis, and the finding of haemorrhage at the time of surgery suggest the inflammatory process became haemorrhagic with treatment. The diagnosis of acute compartment syndrome was suggested by the patient's worsening pain and recorded by measuring compartment pressures. Surgical intervention to prevent permanent disability was required.

Acute compartment syndrome secondary to a ruptured Baker's cyst has not previously been reported, as far as we know. As a ruptured Baker's cyst can mimic a deep vein thrombosis and even predispose to it an exact diagnosis is critical. Our case and the reports of calf haematomas occurring either spontaneously ${ }^{6}$ or after anticoagulation ${ }^{7}$ in patients with ruptured cysts highlight the potential complications of anticoagulation in these patients. The risk of developing a compartment syndrome after a ruptured Baker's cyst, particularly if associated with a coagulopathy, should always be considered. ${ }^{35}$

An acute compartment syndrome is a medical emergency. Irreversible changes are known to occur after only eight to 12 hours of increased compartment pressure, ${ }^{8-10}$ making early diagnosis essential. A history of pain associated with the clinical findings of paraesthesias, weakness, swelling, and increased pain with passive stretching should alert the doctor to the possible diagnosis. Immediate evaluation should include compartment pressure measurements and, if raised, surgical decompression to prevent permanent deformity.

In summary, we present a patient with a ruptured Baker's cyst, complicated by the development of a posterior compartment syndrome. Before the start of treatment in such patients a definitive diagnosis should be made, which often requires both a venogram and an arthrogram. Finally, in patients with increasing pain consideration should be given to the development of an acute compartment syndrome.

1 Adams R. Arthritis, chronic rheumatic, of the knee joint. Dublin Fournal of Medical Science 1840; 17: 520-3.

2 Baker WM. On the formation of synovial cysts in the leg in connection with disease of the knee joint. St Bartholomew's Hospital Reports 1877; 13: 245-61.

3 Wigley R D. Popliteal cysts: variations on a theme of Baker. Semin Arthritis Rheum 1982; 12: 1-10.

4 Wolfe R D, Colloff B. Popliteal cysts: an arthrographic study and review of the literature. $\mathcal{F}$ Bone foint Surg [Am] 1972; 54: 1057-63.

5 Katz R S, Zizic T M, Arnold W P, et al. The pseudothrombophlebitis syndrome. Medicine (Baltimore) 1977; 56: 151-64. phlebitis syndrome. Medicine (Baltimore) 1977 ; 56 : $151-64$.
olff J P, Smiley R H. Spontaneous hematoma of the calf. $\mathcal{f}$ Okla State Med Assoc 1966; 59: 619-21.

7 Wigley R D, Paterson D P. Calf hematoma following anticoagulants in synovial rupture of the knee. NZ Med $\mathscr{f}$ 1982; 95: 630-2.

8 Hargens A R, Schmidt D A, Evans K L, et al. Quantification of skeletal-muscle necrosis in a model compartment syndrome. I Bone foint Surg [Am] 1981; 63: 631-6.

9 Hargens A R, Romine R S, Sipe J C, Evans K L, Mubarak $S \mathrm{~J}$, Akeson $\mathrm{W} \mathbf{H}$. Peripheral-nerve conduction block by high muscle compartment-pressure. $\mathcal{F}$ Bone foint Surg [Am] 1979; 61: 192-200.

10 Sheridan G W, Matsen F A III. Fasciotomy in the treatment of the acute compartment syndrome. $\mathcal{F}$ Bone foint Surg [Am] 1976; 58: 112-4. 